\title{
Wissensbegriff - Weltanschauungsform. Ernst Jüngers epistolare Autorschaft in der Tradition gelehrter Korrespondenz
}

\section{Epistolare Autorschaft und epistemische Geltung: Jüngers nachlass- und werkpolitische Strategien}

Will man sich ein Bild davon machen, welche Rolle Briefe im Hause Jünger spielten, so kann man sich zunächst an die Erinnerungen seines Sekretärs Heinz Ludwig Arnold halten. Dessen Aufgabengebiet umfasste auch die Betreuung der umfangreichen Korrespondenzen des Autors:

\footnotetext{
Das Ordnungssystem war einfach: alphabetisch. Prominenz wurde nicht separat abgelegt - Hitler lag friedlich zwischen unbekannten: Hists - Hits. Auch ein einzelner Brief erhielt sein Papiermäppchen, auf dem der Name des Korrespondenten notiert war. Viele solcher Papiermäppchen wurden in einzelnen Jurismappen gesammelt. Umfangreiche Korrespondenzen füllten allein ganze Jurismappen. Als ich im April 1961 nach Wilflingen kam, waren anderthalb Schränke voll, als ich ein paar Jahre später zum letzten Male meines Ämtchens waltete, war ein ganzes Zimmer mit neuen Schränken auf Zuwachs eingerichtet, bei Jüngers Tod muß es überfüllt gewesen sein. ${ }^{1}$
}

Die schiere Menge allein ist zwar noch kein Indikator für die Bedeutung, die Jünger brieflicher Kommunikation zumaß; die archivarische Akkuratesse, mit der er diese Form des Austauschs bedachte, hingegen schon. Tatsächlich spielen Briefe hinsichtlich des autorschaftlichen Selbstverständnisses Jüngers eine kaum zu überschätzende Rolle. Das betrifft nicht nur den Umstand, dass Teile der Briefwechsel in den veröffentlichten Schriften, vor allen Dingen in den Tagebüchern, auftauchen und dem Briefeschreiben auch im erzählerischen Werk eine thematisch-motivische, vielleicht sogar poetologische Bedeutung zukommt. Bezeichnend ist zudem die Voraussicht, mit der Jünger vorging und für die Detlev Schöttker den treffenden

1 Arnold 2012, 27.

Norman Kasper, Martin-Luther-Universität Halle-Wittenberg

Ә Open Access. (c) 2021 Norman Kasper, publiziert von De Gruyter (c))BY-NC-ND Dieses Werk ist lizenziert unter der Creative Commons Attribution-Non-Commercial-NoDerivatives 4.0 International Lizenz. https://doi.org/10.1515/9783110712568-016 
Begriff der ,Archivautorschaft' reserviert. ${ }^{2}$ Was den ,Archivautor' auszeichnet, ist ein ausgeprägtes ,Nachlassbewusstsein“. ${ }^{3}$ Seine archivarischen Mühen (oder die seiner Sekretäre) schielen nicht nur auf das Interesse der Zeitgenossen; vielmehr schafft der ,Archivautor' die dokumentarische Grundlage für die Befassung mit seinem Werk und seiner Person durch folgende Generationen. ${ }^{4}$ Jüngers Briefwechsel entwickeln sich damit einerseits - insofern sie ediert oder zumindest zitiert werden - zum Teil seines Werks; andererseits stecken sie jenen Deutungs-Horizont $\mathrm{ab}$, in dem bereits vorliegende Werkteile interpretatorisch neu vermessen, korrigiert oder bestätigt werden. Werkpolitik und Nachlasspolitik lassen sich in diesem Zugleich von schleichender ,Ver-Werkung und einem durch das nachlasspolitische Engagement erarbeiteten Statut interpretatorischer Direktiven nicht strikt voneinander trennen, ${ }^{5}$ zumal Jünger zu jenen „Autoren der Moderne“ $\mathrm{zu}$ rechnen ist, die in „einer Epoche der zunehmenden Antizipation des Nachlasses arbeiten." ${ }^{6}$ Eine potentielle philologische Indienstnahme ist hier schon in gleichem Maße Teil der Briefarchiv-Konzeption wie auf die schrittweise In-Werk-Setzung der Korrespondenzen geschielt wird. ${ }^{7}$

2 Schöttker 2016, 112.

3 Vgl. Sina u. Spoerhase 2013; Sina 2017. Vgl. zur Speicherdimension des Archivs Fohrmann 2002. 4 „Archivautoren [...] arbeiten nicht nur für eine zeitgenössische Öffentlichkeit, sondern für spätere Generationen, so dass ihr Werk ohne Berücksichtigung des Archivs als unvollständig zu bezeichnen wäre." Schöttker 2016, 112.

5 Einerseits ist klar, dass als Nachlass das zu gelten hat, „was zu Lebzeiten des Autors keine Werkförmigkeit erlangt hat, und damit folglich nicht Teil einer autorschaftlich gesteuerten Werkpolitik geworden sein kann.“ Andererseits jedoch zielt das nachlasspolitische Wirken zweifelsohne darauf, die Wirkung des Werks zu beeinflussen, so dass „die Nachlasser nicht selten versuchen, durch die Gestaltung ihres Nachlasses ihre Verfügungsgewalt auf die Zeit nach ihrem Ableben auszuweiten. Die Werkpolitik wird von einer Nachlasspolitik flankiert.“ Sina u. Spoerhase 2013, 622.

6 Sina u. Spoerhase, 621.

7 Diese von mir im Anschluss an die nachlasstheoretischen Überlegungen von Detlev Schöttker, Kai Sina und Carlos Spoerhase formulierten Überlegungen zu Jünger bedürfen weiterer Diskussion, die das epistolare Interesse des Beitrages überschreiten würde. Es sei an dieser Stelle nur am Rande vermerkt, dass innerhalb der Jünger-Forschung keine Einigkeit darüber herrscht, inwieweit nachgelassene Briefe und Manuskripte tatsächlich, wofür Schöttker und ich plädieren, als Teile des Werkes zu gelten haben. So kritisiert Joanna van de Löcht kürzlich die sich ganz auf Jüngers florale Manuskript-Einklebungen konzentrierende Studie von Sibylle Benninghoff-Lühl Die ganze Welt ein Garten? Flora und Fauna in Ernst Jüngers schriftlichem Nachlass mit dem Hinweis darauf, die Autorin nutze die „Figur eines hypothetischen Lesers, der die Manuskriptseiten mit ihren Einklebungen rezipiert“: „Außer Acht gelassen wird hierbei, dass die Manuskripte eben keine öffentlichen Dokumente sind, sondern nur für die private Nutzung gedacht waren." Van de Löcht 2020, 229. Die Feststellung will deutlich machen, dass es unzulässig sei, nichtveröffentlichte Ma- 
Überschaut man die forschungsgeschichtlich nachweisbaren Grundlinien im Umgang mit Jünger, so überrascht es nicht, dass es bisher vornehmlich als gesellschaftspolitisch und zeitgeschichtlich relevant eingeschätzte Aspekte seiner Korrespondenzen sind, die im Mittelpunkt des Interesses stehen. Die Briefpartner Martin Heidegger, Carl Schmitt, Friedrich Hielscher, Gerhard Nebel, Ernst Niekisch und - kürzlich entdeckt und zugänglich gemacht - Joseph Wulf sind nur einige der Autoren, die die Auseinandersetzung mit Jünger maßgeblich prägen. ${ }^{8}$ In Aussicht gestellt werden hier authentische Antworten auf Fragen nach Jüngers Verhältnis zu Gewalt, Nationalismus, Faschismus, Antisemitismus, Humanismus, Nihilismus und Demokratie. Dieses Frageinteresse knüpft an ältere Debatten um Jüngers ,Wandlung 'vom nationalistischen Heros der Gewalt zum kunstsinnig-seherischen Europäer an und stellt sie auf eine Quellengrundlage, die verlässliche Auskünfte verspricht. Dabei ist es entweder aus dem Blick geraten oder man hat es schlichtweg nicht akzeptiert und als Finte gedeutet, dass Jünger nach 1945 (eigentlich bereits seit 1933) nicht mehr in erster Linie als politischer Autor in Erscheinung tritt. Sein Versuch, sich einen metahistorischen Betrachtungsstandpunkt zu erarbeiten, mündet spätestens dann mit der Vorbereitung der ersten Werkausgabe um 1960 in die Konzeption einer musischen Autorschaft und die autorschaftliche Selbstreflexion entwickelt sich zum Teil des Werks. Verbunden ist dies nicht nur mit der Hinwendung Jüngers zu geologischen, naturgeschichtlichen und archäologischen Themen, sondern auch mit dem systematischen Aufbau eines Briefarchivs. ${ }^{9}$ Für diejenigen, die einen entpolitisierten Jünger nicht gelten lassen wollten, war klar: Erdgeschichtliche und kulturgeschichtliche Themen sind bei diesem Autor nur insofern von Belang, als diese im Fokus einer Veränderung der radikalen politischen Haltung Jüngers, die er in den 1920er und frühen 1930er Jahren einnahm, und damit als Variation gesellschaftspolitisch konturierter Autorschaft betrachtet werden. Daniel Morat spricht hier von der Entpolitisierung des Politischen und meint damit die Verhandlung von „im Kern politischen Themen“ im Duktus der „metapolitischen und posthistorischen Reflexion“10. In dieser Perspektive gilt Jün-

nuskriptteile so zu lesen und zu betrachten, als ob sie veröffentlicht wären. An Benninghoff-Lühls assoziativ-verdächtigender Deutung Jüngers ist sicherlich vieles problematisch; die präsupponierte Werkförmigkeit der Manuskripte jedoch nicht, wird diese doch Jüngers ,Archivautorschaft‘ gerechter als eine Trennung in Privatheit und Öffentlichkeit, die zu einer konzeptionellen Klärung von Jüngers ,Nachlassbewusstsein“ nichts beiträgt.

8 Vgl. die jeweiligen Einträge im Ernst Jünger-Handbuch; zu Heidegger: Trawny 2014; zu Schmitt: Villinger 2014; zu Hielscher: Schmidt 2014; zu Nebel: Neumann 2014; zu Niekisch: Morat 2014. Den Briefwechsel Jüngers mit Joseph Wulf legte Schöttker 2019 vor.

9 Vgl. Schöttker 2016, 112-121.

10 Morat 2012, 179. 
gers erdgeschichtlich-planetarische wie auch die archäologisch-vorgeschichtliche Ausrichtung als ,esoterische Position“, ${ }^{11}$ genauer gesagt: als Camouflage einer eigentlich gesellschaftspolitischen Orientierung, die es zu enträtseln und zu vorhergehenden Stationen des politischen Engagements in Beziehung zu setzen gelte. Gegenüber dieser primär politisch-ideengeschichtlich konturierten Entwicklungslogik, so meine ich, muss es darum gehen, die ,esoterischen' Wissensfelder Jüngers im Einzelnen genauer zu vermessen, bevor sie auf ihre möglichen politischen Implikationen hin - und damit auch eine genauere Konturierung dessen, was sich sinnvoller Weise als ,politisch` ansprechen lässt - befragt werden können. Briefwechsel aus Jüngers Archiv, die in dieser Hinsicht Aufschluss versprechen, wurden bisher jedoch weder systematisch identifiziert noch wissensgeschichtlich ausgewertet. Das wäre aber notwendig. Denn es ist in der Tat überraschend, dass Jünger mit Wissenschaftlern unterschiedlicher Disziplinen im brieflichen Kontakt stand, Fachfragen diskutierte, Geoden wechselte sowie Publikationen austauschte und kommentierte. Ich habe an anderer Stelle einige dieser Paläontologen, Geologen und Anthropologen im brieflichen Gespräch mit Jünger vorgestellt und im wissensgeschichtlichen Kontext ihrer Zeit verortet. ${ }^{12}$

Diese Briefe und die sie begleitenden Objekte lassen sich in mancherlei Hinsicht als Teil eines Gelehrtendiskurses, genauer gesagt: als gelehrte Korrespondenz ansprechen. Der Beitrag möchte den Zusammenhang von Jüngers epistemischepistolarer Autorschaft mit der gelehrten Tradition etwas genauer beschreiben. Dazu ist es in einem ersten Schritt notwendig, die Rolle, die er in seinen naturgeschichtlichen Briefen einnimmt, als ,Autor-Funktion“ (Foucault) zu konkretisieren. In dem Maße, wie Jüngers epistemischer Selbstentwurf durch die Wissensperspektivierungen seines ,Brief-Ichs' Konturen gewinnt, kann es nicht darum gehen, durch dieses ,Brief-Ich` die Person Jünger selber sprechen zu hören. Zu analysieren ist der Autor vielmehr als Funktion im Brief-Text. Ich werde dabei auf den - zugegeben: nicht unumstrittenen und nicht immer aussagekräftigen - Begriff der ,Inszenierung“ zurückgreifen. Die Rede von der ,Ich-Inszenierung‘ soll hier das konstruktive Moment der autorschaftlichen Rollenbildung als epistemische Autorschaft markieren. Als Bezugspunkt eines solchen Austauschs von Wissen und der Diskussion von Wissenshorizonten bietet sich ein Blick auf den gelehrten Brief an. Liest man gelehrte Briefe, und hier besonders jene naturwissenschaftlichen Inhalts des 17. und 18. Jahrhunderts, als „Handlungsdispositive, die zur Steuerung und Stabilisierung der Erwartungen der jeweils Kommunizierenden

11 Morat 2012, 180.

12 Vgl. Kasper 2021. 
dienen“13, dann ist damit genau jener Punkt angesprochen, der auch für Jüngers epistemische Autorschaft maßgeblich ist. Als Briefschreiber entwirft und streut er jenes Wissens- und Weltanschauungsideal, das auf die Wahrnehmung anderer Textsorten des eigenen Werks abfärbt und seine Determinationskraft vor allem aus der Tatsache bezieht, dass es im Austausch mit etablierten, bisweilen disziplinär institutionalisierten, auf jeden Fall sachkundigen, wenn man so will: gelehrten Briefpartnern gewonnen wird.

\section{Inszenierter Anti-Darwinismus. „Autor-Funktion“ und Brief-Ich zwischen Wissen und Weltanschauung}

Einer dieser Briefpartner ist der Anthropologe und Evolutionsbiologe Gerhard Heberer (1901-1973). Jünger stand mit ihm von den 1950er Jahren bis Mitte der 1960er Jahre in brieflichem Kontakt. Seit den 1940er Jahren vertritt Heberer, etwa in der vielrezipierten, von ihm herausgegebenen Evolution der Organismen (1943), später auch in anderen, teilweise in Jüngers Wilflinger Bibliothek nachweisbaren Publikationen, eine am Evolutionsdenken Darwins geschulte, durch Ergebnisse der modernen Genetik konkretisierte Variante der Deszendenztheorie. ${ }^{14}$ In Abkehr und Kritik von Typensprunglehren, die neue Arten als ,Schöpfungen“ verstehen, geht es Heberer um den Nachweis, dass biologische Gestaltbildungsprozesse Fortsetzungen und Veränderungen bereits bestehender Lebensformen sind. Stammesgeschichtlich betrachtet also ist die Annahme von ,Typen‘, die einander ablösen würden, abzulehnen. Stattdessen müsse von unzählig vielen, kaum beobachtbaren Veränderungsschritten ausgegangen werden, die in der Summe, d.h. über Jahrmillionen hinweg, sichtbare Veränderungen zeitigen. Hauptgegner eines solchen Denkens sind neo-lamarckistische und orthogenetische Theorien, die die Eigengerichtetheit der Entwicklung der Lebensformen betonen und äußeren Umwelt-Einflüssen - wie sie Darwin herausstellt - keinerlei Relevanz zugestehen wollen. Jünger übernimmt im Briefwechsel die Rolle des Verteidigers anti-darwinistischen Denkens. Im Kern geht es ihm dabei um die Annahme einer der zeitlichen Entwicklung entzogenen Prägekraft (paläo-)biologischer Formbildungsprozesse. So kritisiert er etwa in einem Brief an Heberer einen „biologischen Philosophen oder besser wohl philo-

13 Kempe 2004, 411.

14 Vgl. Kasper 2021, 86-94. 
sophierenden Biologen“15 für dessen Versuch einer ,philosophischen` Deutung der Deszendenztheorie. Gemeint sein dürfte damit wohl der Wissenschaftstheoretiker Hugo Dingler (1881-1954), ein Beiträger der Evolution der Organismen, ${ }^{16}$ dem nach seinem Tod - 1956 - ein „Gedenkbuch“ gewidmet wird, an dem sich Heberer mit dem Aufsatz Die Stellung Hugo Dinglers zur Evolutionstheorie beteiligt. ${ }^{17}$ Jünger wiederum nimmt in seinem Brief an Heberer, in dem er die „Erinnerungsschrift“ erwähnt, auf diesen, mutmaßlich Dingler gewidmeten Beitrag Heberers wie auch Dingler als Deszendenzphilosoph Bezug:

Nicht überzeugt haben mich die Ausführungen des biologischen Philosophen oder besser wohl philosophierenden Biologen, dem ihre Erinnerungsschrift gewidmet ist. Unter seinen Argumenten findet sich nicht eines, das jenseits der physikalischen-biologischen Sphäre liegt, er unterstützt die Naturwissenschaft mit ihren eigenen Hilfskräften. Ein Denkvermögen, das sich über die Idealität der Zeit nicht wenigstens annähernd Rechenschaft geben kann, kommt von dem Rade des Ixion nicht ab. [...] Wo Naturwissenschaftler sich philosophisch oder theologisch bemüht haben, ist es meist schief gegangen, und offenbar auch hier. Der Ort, an dem das Puzzle-Spiel mit unerhörter Intelligenz und oft auch Divination zusammengesetzt wird, ist ein anderer als jener, an dem es in toto vorliegt und in die Mahlgänge der Zeit geworfen wird, die es zerstückt. Dies gilt auch für die Menschwerdung. ${ }^{18}$

Dingler liegt ganz auf der Linie Heberers, wenn er allein physikalischen und chemischen Komponenten in evolutionstheoretischer, anthropogenetischer Hinsicht einen Erklärwert zuerkennen will, vitalistischen Parametern wie auch Typensprungtheorien hingegen skeptisch gegenübersteht. ${ }^{19}$ Heberer verteidigt seinerseits Dingler in der von Jünger angesprochenen „Erinnerungsschrift“ mit allen Kräften. Entscheidend für das Verständnis der Konfliktlinie Jünger vs. Heberer/Dingler ist zweifelsohne der Umstand, dass die von Jünger angemahnte Berücksichtigung der „Idealität der Zeit“ - eigentlich eine Aufforderung zur neuplatonischen Grundierung stammesgeschichtlicher Prozesse - weder von Heberer noch von Dingler unterstützt wird. Ohne an dieser Stelle weitere wissensgeschichtliche Details zu referieren, soll eine Frage angeschlossen werden, die versucht, das epistolarepistemische Profil Jüngers etwas genauer zu bestimmen: Welche Funktion kommt dem Autor zu, der aus Jüngers Briefen spricht? Was heißt es also, wenn sich das Ich des Briefes hier als „,[n]icht überzeugt“ zeigt? Oder zunächst grundsätzlicher gefragt: Welcher Status kommt diesem Ich argumentationslogisch überhaupt zu?

15 Jünger an Heberer, 18. Juli 1956. Nachlass Ernst Jünger, DLA Marbach.

16 Vgl. Dingler 1943, Dingler 1954.

17 Heberer 1956.

18 Jünger an Heberer, 18. Juli 1956. Nachlass Ernst Jünger, DLA Marbach.

19 Vgl. Gutmann 2006. 
In der Briefforschung hat sich eine Sichtweise auf das Brief-Ich etabliert, die dessen inszenatorischen Charakter herausarbeitet und davor warnt, es umstandslos mit der Person des Autors selbst zu identifizieren. Eine derartige Betonung des ,inszenatorisch-fiktionalen Potential[s]“20 brieflicher Rede hat Robert Vellusig kürzlich kritisiert und eine Schärfung der Begriffe ,Inszenierung‘ und ,Fiktion` angemahnt; würden diese doch einem Unaufrichtigkeitsverdacht gegenüber dem Briefschreiber das Wort reden, der im Ergebnis epistolare Modi „personaler Selbstdarstellung“ generell mit „,Scheinhaftigkeit““21 assoziiere. So nachvollziehbar (und damit ernst zu nehmen) dieser Kritikpunkt hinsichtlich einer Erörterung der narrativen Verfasstheit personalen Erscheinens in Privatbriefen ist, so lässt er sich doch nicht einfach auf das Brief-Ich gelehrter Korrespondenzen übertragen, denn hier steht weniger die Opposition , authentisch ${ }^{\text {- }}$, nicht authentisch ${ }^{`}$ zur Diskussion als die Rolle des Brief-Ich zum präsentierten Wissen. Fundiert man dieses Brief-Ich wie es im Folgenden unternommen werden soll - in Foucaults ,Autor-Funktion', so löst man die polarisierende Entgegensetzung von Inszenierung/Fiktionalität und authentischer Selbstpräsentation auf und öffnet das inszenatorische Potential hin zu einer Konkretisierung der autorschaftlichen Rolle im Text, genauer gesagt: hin zu einer epistemischen Autorschaft. Unter epistemischer Autorschaft verstehe ich nicht einfach nur den Austausch und die Kenntnisnahme von Wissen; es geht vielmehr um Modi der Diskussion, Perspektivierung, Übernahme, Anverwandlung oder Kritik von (wissenschaftlichen) Wissensbeständen. Meine These ist hier, dass das inszenatorische Potential des Ich in den Nachlass-Briefen Jüngers ganz maßgeblich darauf abzielt, eine bestimmte epistemische Autorschaft zu befördern, eine Autorschaft nämlich, die auch die Konstitution und leserseitige Wahrnehmung und Anerkennung epistemischer, d.h. hier: naturphilosophischer, geologischer und paläobiologischer Expertise bezüglich anderer Teile des Werks zu fundieren sucht.

Nach Foucault ist es ein wichtiges Merkmal der „Funktion Autor“, „dass sie als das „Ergebnis einer komplizierten Operation“ betrachtet werden muss, die ein „gewisses Vernunftwesen, das man Autor nennt“22, hervorbringt. Was an einem „Individuum als Autor“ angesprochen wird, ist im Ergebnis die „mehr bis minder psychologisierende Projektion der Behandlung, die man den Texten angedeihen läßt, der Annäherungen, die man vornimmt, der Merkmale, die man für erheblich hält, der Kontinuitäten, die man zuläßt, oder der Ausschlüsse, die man macht.“23

20 Schuster/Strobel 2012, XIII.

21 Vellusig 2018, 61. Vgl. auch Vellusig 2020, 210-212.

22 Foucault 1988, 20. Vgl. zu Foucaults Annahme, der Autor sei ein konstruiertes Vernunftwesen, Jannidis 1999, 356-360.

23 Foucault 1988, 20. 
Auf diesem Weg entsteht der Autor als „Feld eines begrifflichen und theoretischen Zusammenhangs“24. Gestiftet wird der Zusammenhang maßgeblich durch den Leser und insofern ist die „Autorfunktion in erster Linie eine Rezeptionskategorie.“25 Dies entbindet jedoch nicht davon, den „Ort innerhalb des Diskurses, von dem aus das Subjekt sich äußert“, ${ }^{26}$ genauer zu vermessen, wird doch hier seine Funktion als Autor für den Rezipienten greifbar. Insofern ist eine Analyse der Autor-Funktion keineswegs notwendig mit der Abschaffung der Kategorie des Autors verbunden. ${ }^{27}$

Blickt man von Foucault aus kommend auf das Brief-Ich Jüngers, so muss es darum gehen, epistemische Autorschaft als Feld des durch die „Autor-Funktion“ gestifteten begrifflichen und theoretischen Zusammenhangs lesbar zu machen. Der Autor interessiert als „Instanz der Zuschreibung sprachlichen und kulturellen Wissens“, ${ }^{28}$ wir wollen hinzufügen: auch wissenschaftlichen Wissens. Die Teilhabe des Autors am Fachgespräch, seine epistolare Einschreibung in den diskursiven Fach-Zusammenhang bilden hier die maßgebliche Grundlage dafür, die Diskussion anderer, im gleichen Zeitraum entstandener Werkteile im Rahmen jenes begrifflichtheoretischen Zusammenhangs führen zu können. Man kann dies durchaus als Versuch einer nachlassbasierten „Selbsthistorisierung“29 Jüngers begreifen. Sieht man den Nachlasser Jünger nämlich in der Position dessen, der sich, wie es Kai Sina und Carlos Spoerhase für die „Selbsthistorisierung“ für maßgeblich erachten, bereits zu Lebzeiten , als einen möglichen historischen Gegenstand späterer Nachforschungen versteht“", ${ }^{30}$ dann rücken jene Kontexte - und damit Kontext-Texte in den Mittelpunkt, die der Autor hinsichtlich der Würdigung seines Werks für maßgeblich erachtet oder zumindest als maßgeblich erachtet wissen will. Die Kontexte, die für eine Auslegung des Werkes Jüngers heranzuziehen sind, erhalten durch die Briefe ihre Konturen, werden gleichsam präfiguriert. Simone Winko hat auf die epistemische „Funktion des Autorbezugs“ in der Auslegungspraxis hingewiesen: „Hier geht es um die Sicherung der in die Interpretation einzubeziehenden Kontexte“, wobei mit dem „Kriterium ,Wissen des Autors“ Typen von Kontexten unterschieden“ ${ }^{31}$ werden. „Immer noch am häufigsten werden Kontexte einbezogen, die der Autor gekannt, geschätzt, absichtlich aufgenommen, modifiziert oder weitergeführt hat“;

24 Foucault 1988, 21.

25 Spoerhase 2007, 53.

26 Spoerhase 2007, 55.

27 Vgl. Jannidis 1999, 358.

28 Schaffrick/Willand 2014, 31.

29 Vgl. Sina u. Spoerhase 2013, 612-617, 623; Sina 2017, 52-58.

30 Sina u. Spoerhase 2013, 623.

31 Winko 2002, 345. 
in unserem Fall handelt es sich um ,wissenschaftliche Ideen oder Erkenntnisse“, 32 die den Kontext abgeben. Entscheidend ist dabei: Jüngers Brief-Archiv erschafft jenen Kontext-Kosmos, an dem die argumentative Verwendung des ,Autorbezuges Jünger‘ in der interpretatorischen Praxis nicht vorbeikommt, denn die Briefe konstituieren jenen hermeneutischen Bezugsraum, der dem Leser Einblicke in die Genese seiner naturphilosophischen Positionen als potentiell relevante, auf jeden Fall bei der Deutung seines Werkes zu berücksichtigende Kontexte ermöglicht. Während diese Positionen - gleichsam als ,Spitze des Eisbergs“ - sein veröffentlichtes Werk prägen, liefern die (nur in Teilen veröffentlichten und zitierten) Briefe Einblicke in Denkbewegungen und abgelehnte wie auch präferierte Bezugstheorien, deren Kenntnisnahme, Prüfung und Bewertung dokumentarisch ausgestellt wird und zum Ermitteln weiterer Kontext-Texte (etwa von Dingler oder Heberer) auffordert. So ist es auch hinsichtlich des oben zitierten Briefs an Heberer. Das Brief-Ich Jüngers konstituiert dabei erst maßgeblich jene epistemische Autorschaft, die dann von außen interpretativ in Anspruch genommen werden kann, wenn es darum geht, Kontext-Wissen zu identifizieren und zum veröffentlichten Werk in Bezug zu setzen. Interessant ist dabei hinsichtlich der Kommunikationsform (Nachlass-)Brief und der damit in Rechnung gestellten Selbsthistorisierungsabsichten Jüngers: Der eigentliche, zumindest wichtigere Adressat dieses Brief-Ichs wäre wohl nicht der Anthropologe Heberer - sondern der Leser Jüngers, denn dieser ist es nämlich, der in der Zusammenschau von Briefen, Erzählungen und Essays, um es mit Felix Vodička zu sagen: „Autor-Konkretisation“33 betreiben soll, und zwar in Richtung epistemischer Autorschaft. Vodička denkt dabei an den Autor in seiner „metonymische[n] Bedeutung, an die Einheit, die die Werke eines bestimmten Autors in ihrer Gesamtheit bilden““.34 Jüngers Brief-Ich gibt hinsichtlich der zu leistenden, nachlass- und werkpolitisch forcierten ,Konkretisation“ wichtige Hinweise.

An dieser Stelle sollen wenige kurze Bemerkungen zu einem interpretativen Umgang mit Jüngers Texten und einer „Autor-Konkretisation“ im Zeichen der Inszenierung epistemisch-epistolarer Autorschaft genügen. Die Kritik an der Zeitkonzeption des ,Darwinismus‘, verbunden mit der Annahme idealer, qualitativ anders verfasster Zeit, ist spätestens seit Heliopolis (1949) in Jüngers Werk zu einem

32 Winko 2002, 345.

33 „Wenn wir ein neues Werk eines Autors lesen, der uns bereits aus manchen seiner früheren Werke bekannt ist, so nehmen wir ganz instinktiv übereinstimmende oder auch von früherer Erfahrung abweichende Zeichen wahr, und alle erfaßten Eigenschaften [...] bilden eine verallgemeinerte, aus dem Werk hervorgehende, jedoch außerhalb des Werks existierende Autor-Funktion." Vodička 1976, 114.

34 Vodička 1976, 114. 
Topos geronnen, der nur mit wenigen Worten und Symbolen - wie dem titelgebenden Zeitmesser im Sanduhrbuch (1954) - aufgerufen werden muss, um sich argumentativ geltend zu machen. Wie intensiv Jünger sich mit naturgeschichtlichen Detailfragen befasst, bleibt einigen Lesern nicht verborgen, so etwa Hermann Hesse, der in seiner Besprechung (1960) von Jüngers An der Zeitmauer (1959) „das reiche Material aus der Geologie, Paläontologie, Zoologie und anderen Disziplinen der Naturwissenschaften, das der Verfasser zusammenträgt“" ${ }^{35}$ erwähnt. Der Brief an Heberer legt nun nahe, dass Jünger es bei dem Zusammentragen des Materials nicht bewenden ließ; darüber hinaus tritt er in ein Gespräch ein, das seine Positionen in Konfrontation mit anderen (Heberer, Dingler) konturiert. Entscheidend ist dabei weniger das konfrontative Moment (von Jünger erwartet man diesbezüglich auch nichts anderes), als vielmehr die der Gelehrtenrolle nicht unähnliche zur Schau gestellte Fähigkeit der Rezeption und Diskussion bestehenden naturwissenschaftlich-naturphilosophischen Wissens, das gleichsam den breiten Kontext modelliert, in dem er wahrgenommen zu werden wünscht. Es ist der Brief als potentiell an Jüngerleser adressierte (und nicht empfängeradressierte) Kommunikationsform, der die Inszenierung einer solchen epistemischen Autorschaft ermöglicht. Vom Brief aus lassen sich Aufsätze und Bücher identifizieren, die Jünger mit hoher Wahrscheinlichkeit zur Kenntnis genommen haben könnte; und von einer dem Brief-Ich Jüngers zugewiesenen Intention können mögliche Lesarten dieser Aufsätze und Bücher rekonstruiert werden. Dabei ist die Überschreitung des wissenschaftlichen Wissens hin zum seherischen (und damit eben nicht mehr wissenschaftlichen) Idealismus der Schau entzeitlichter Gestalten auf die Kenntnisnahme dieses wissenschaftlichen Wissens angewiesen, geht es doch um den Nachweis ,falschen' Zeitbewusstseins. Erst wenn dieser gelingt, kann die Entgrenzung in die Zeitlosigkeit als divinatorische Alternative auftreten: „Der Ort, an dem das Puzzle-Spiel mit unerhörter Intelligenz und oft auch Divination zusammengesetzt wird, ist ein anderer als jener, an dem es in toto vorliegt und in die Mahlgänge der Zeit geworfen wird, die es zerstückt.“36 An der Rekapitulation des wissenschaftlich sanktionierten Wissens führt jedenfalls kein Weg vorbei, auch wenn es (nur) darum geht, dessen Begrenztheit vor Augen zu führen und damit den Punkt zu markieren, an dem ,unerhörte[] Intelligenz und oft auch Divination“ als maßgeblich erachtet werden: „Die Zeit ist der Funke, der für unsere Blicke das Feuerwerk in Bewegung zu setzen scheint. Das ist ein Augentrug. Daran ändert nichts, daß wir die Bahnen und die farbigen Äste der Rakete messen und in

35 Hesse 2005, 352.

36 Jünger an Heberer, 18. Juli 1956. Nachlass Ernst Jünger, DLA Marbach. 
Relation bringen können, aber hier ist die Naturwissenschaft an ihrem Platz. “37 Die Weltanschauungsform ist auf eine Rekonstruktion des wissenschaftlichen Argumentationshorizontes („Naturwissenschaft“) und dessen Zeitverständnis angewiesen, will sie nicht als bloßer Mystizismus erscheinen. Freilich lässt sich das divinatorische Moment selbst kaum begrifflich einholen; markiert wird vielmehr ein figürliches, d.h. an Denkfiguren orientiertes Denken, in dem der Begriff zur Figur, der Wissensbegriff zur Weltanschauungsform wird. Denkfiguren in diesem Sinne stellen, wie Jutta Müller-Tamm im Anschluss an Erich Auerbachs FiguraAufsatz (1938) formuliert, einen ,beweglichen - übertragbaren, zirkulierenden - und wandelbaren Vorstellungszusammenhang im Prozess des Denkens, der Weltaneignung oder der Zeichenproduktion“38 dar. In epistemologischer Hinsicht kommt der Denkfigur eine welterschließende Funktion zu, die sie mit der ,konzeptuellen Metapher‘ (George Lakoff, Mark Johnson) verbindet. ${ }^{39}$

\section{Information und Streit - gestern und bei Jünger}

Rücken wir Jüngers epistolare Autorschaft in die Tradition des Gelehrtenbriefs ein, so muss man sich zunächst klar machen, dass seine autorschaftliche Rolle zu keinem Zeitpunkt die eines Wissenschaftlers ist. Gleichwohl indizieren die „Attribute ,wissenschaftlich“ oder ,gelehrt““ auch mit Blick auf Jüngers Korrespondenzen, wie es Michael Kempe für den frühneuzeitlichen Gelehrtenbrief grundsätzlich für maßgeblich erachtet, „dass es hier weniger um die Vermittlung von Emotionen oder Glaubensaussagen, als vielmehr um die Übertragung - dem Anspruch nach systematisierbarer Informationen (gleich welcher Art der zugrundegelegte Wahrheitsbegriff ist)“40 geht. Der epistolare „Transfer von Wissen“, verstanden als Austausch „naturwissenschaftliche[r] Informationen“ zwischen Naturforschern des 17. und 18. Jahrhunderts im Rahmen von „Korrespondenznetze[n]“"41, beinhaltet auch eine Gewichtung und Relationierung der Wissensbestände im Koordinatensystem des zeitgenössischen Wahrheitsverständnisses. Die Gegenstände der „Wissensverhandlung“, so Thomas Wallnig mit Blick auf den lateinischen Gelehrtenbrief des 16. bis 18. Jahrhunderts, „korrelieren mit den zentralen Themen der frühneuzeitlichen Wissenskultur und -entwicklung“:

37 Jünger an Heberer, 18. Juli 1956. Nachlass Ernst Jünger, DLA Marbach.

38 Müller-Tamm 2014, 101.

39 Vgl. Müller-Tamm 2014, 102.

40 Kempe 2004, 411.

41 Kempe 2004, 414. 
Im selben Ausmaß, wie die Korrespondenzen etwa religiös-philosophische Streitfragen, Kopernikanismus und Newtonianismus, Antiquarianismus, Bibelkritik und historisch-kritische Methodologie [...] und experimentelle Physik ins Zentrum rückten, wurden diese Themen zugleich zum ideellen Kernreservoir dessen, was in einem Diskurs der Selbstvergewisserung mit ,Humanismus‘, ,Wissenschaftlicher Revolution‘ und ,Aufklärung' gleichgesetzt wurde und auch heute noch wird. ${ }^{42}$

Einzelne Wissenselemente bekommen hier als Teil eines „,ideellen Kernreservoir[s]“ eine bestimmte Signifikanz hinsichtlich des vertretenen Wahrheitsanspruches und Wahrheitsstrebens zugewiesen, die dann zum Leitbild einer gesamten Epoche erklärt werden. Mit Blick auf den von uns gesetzten Fokus entscheidend ist: Jüngers Auseinandersetzung mit Heberer und Dingler steht in der Tradition einer Streitfragenkultur, die einzelne Wissenssegmente auf ihren Beitrag zu einer ,philosophischen' Wahrheitsfindung hin fokussiert. Es überrascht nicht, dass es deszendenztheoretische Fragen sind, um deren angemessene ,philosophische“ Imprägnierung es geht, reiht sich doch der ,Darwinismus' des 19. Jahrhunderts von Anfang an in einen Fragehorizont ein, der entlang der Konfliktlinie, Schöpfung 'vs. ,Abstammung ' in seiner bipolaren Codierung und einem damit einhergehenden dezisionistischen Apriori älteren Streitkonstellationen folgt und - besonders um 1900 - ein enormes weltanschauliches Potential zugemessen bekommt. Trotz aller Unterschiede im Detail ist es sicher nicht falsch, Jüngers Verteidigung der biologischen ,Schöpfung 'als geprägte, der zeitlichen Entwicklung entzogene Formidee in der Tradition des naturphilosophisch-weltanschaulichen Denkens eines Wilhelm Bölsche oder Ernst Haeckel zu sehen, gerade auch in der Verbindung von Theologie und Biologie. Berücksichtigen muss man zudem, dass Spielarten einer solchen ,idealistischen Morphologie‘ Mitte des 20. Jahrhunderts gleichfalls in der disziplinären Paläontologie durchaus noch vertreten waren, so dass sich Forscher wie Heberer und Dingler damit auseinanderzusetzen hatten (auch wenn sie dem freilich nichts abgewinnen konnten).

Einem Blick, der Jüngers epistolare Autorschaft in der Tradition gelehrter Korrespondenzen betrachtet, bleibt es nicht verborgen, dass zu den Briefen auch fossile Zeugnisse hinzukommen. Gewechselt wurden diese etwa mit Otto Klages und Klaus Ulrich Leistikow. ${ }^{43}$ Man kann hier, um den Bezug zur Tradition einsichtig zu machen, z.B. auf den Fossilienaustausch von Johann Jakob Scheuchzer (Zürich) und John Woodward (London) verweisen. ${ }^{44}$ Anfang des 18. Jahrhunderts gewährten die beiden Gelehrten dem Korrespondenzpartner Einblicke in ihre

42 Wallnig 2020, 475.

43 Vgl. Kasper 2021, 59-86, 94-124.

44 Vgl. Kempe 2004, 418-420. 
Sammlungen, besprachen interessante Funde und arbeiteten vor allem an einer Klassifikation geologischer Zeugnisse. Im Rahmen der regen Versendepraxis gingen die Sammlungen beider bald ineinander über: „Hier zeigt sich der Wandel vom geschlossenen Raritätenkabinett des 17. Jahrhunderts zur offenen, auf wissenschaftlichem Austausch basierenden Naturaliensammlung des 18 . Jahrhunderts. “45 Jüngers Sammlungspraxis kehrt diese Entwicklung gewissermaßen wieder um, wenn er die steinernen Zeugnisse gemeinsam mit diversen Sammlungsstücken unterschiedlicher (z.B. naturkundlicher, ethnologischer, kunstgeschichtlicher) Ordnung und Herkunft (Europa, Asien, Amerika) kombiniert. Pate gestanden haben dürfte hier die barocke Wunderkammer, in der natürliche Objekte und künstlerische Artefakte ein Kuriositätenkabinett bilden. Die „Ammonitenreihe spiegelt“, wie es in einem in Strahlungen III (Siebzig verweht I) aufgenommenen Brief an Otto Klages vom 04.01.1966 angesichts der stammesgeschichtlich nachweisbaren FormEntwicklung fossiler Ammoniten heißt, „den Rhythmus des Universums. “46 Fortgesetzt wird damit die ältere Annahme von Entsprechungsverhältnissen zwischen Mikro- und Makrokosmos, die auch die Präsentation von Ausstellungsstücken im Rahmen von Wunderkammern strukturierte. Doch wozu dient dieser Bezug? Jüngers Deutungshorizont der „Ammonitenreihe“ verweist deutlich darauf, dass es um die Rückeroberung eines ganzheitlichen, vormodernen, voraufklärerischen Erkenntnis- und Wissensanspruchs geht. Dieser wiederum ist typisch für ein Gelehrtenideal, dem ein bestimmtes Autorschaftsverständnis entspricht.

\section{Autorität durch, Wahrheit‘. Vormoderne Autorschaft in der Moderne}

Felix Steiner hat die Unterschiede zwischen einer vormodernen und einer aufgeklärt-modernen Autorschaft herausgearbeitet. Während der naturwissenschaftliche Autor seit dem späten 18. Jahrhundert von der Einsicht geleitet werde, dass seine Texte „nicht ,fertige“ Erkenntnis medial übersetzen und dass die Funktion des Textes auch gar nicht primär in der Darstellung einer präexistenten Welt der Erkenntnis und der Tatsachen bestehen kann, sondern dass Erkenntnis- und Tatsachenkonstruktion im Text hergestellt werden “ ${ }^{47}$ folge der vormodern-frühneuzeitliche Autor einem Impuls, der „wissenschaftliches Wis-

45 Kempe 2004, 418-419.

46 Jünger 1982, 238.

47 Steiner 2009, 60. 
sen“ als „ontologische ,Wahrheit““ auffasst, deren sprachliche Repräsentation gleichsam „autoritär gesicherte Geltungsansprüche“48 formuliere:

Ontologisches Wissen fixiert die Dinge, ,wie sie sind'. Ziel der Wissensgewinnung ist die Beantwortung der Frage: Wie lässt sich die Welt, der Mensch, die Sprache usw. als das beschreiben und erklären, was sie sind? Theorien und Methoden helfen, die wahre Existenz der Dinge zu identifizieren. Wahrheit ist hier nicht ein menschgemachter Annäherungswert, sondern Wahr und Unwahr gleichen einem ausschließlichen Entweder und Oder. Das heisst der Streit um ontologische Gewissheitsansprüche kann ausführliche und komplizierte Formen annehmen, wobei die Differenz zwischen dem Sein der Gegenstände und jenem autorschaftlichen Bewusstsein, das für Attribuierung und Prädizierung aufkommt, konsequent ausgeblendet wird. Tatsachen scheinen für ihre Eigenschaften selbst verantwortlich zu sein, entsprechend erscheint ein ontologisches Wissen immer in einem endogenen Sinne an seinen Gegenstand gebunden. ${ }^{49}$

In der Perspektive Jüngers und der geistesgeschichtlich orientierten Aufklärungskritik der ersten Hälfte des 20. Jahrhunderts gerät jenes ontologische Wissen seit Descartes, spätestens dann seit Kant immer massiver unter Druck. Jüngers autorschaftliche Rolle setzt in dieser Situation ganz darauf, eine „Autor-Funktion“ zu inaugurieren, der ein exklusiver Zugriff auf eine in unterschiedliche Sichtweisen und Aussageweisen zerfallende ,Wahrheit" gelingt. Wir hatten mit Foucault den Autor als „Feld eines begrifflichen und theoretischen Zusammenhangs“"50 gefasst und können nun mit Blick auf den Autor Jünger präzisieren: Das hier in Rede stehende Feld wird ganz maßgeblich durch ein ontologisches Wahrheitsdenken abgesteckt, dem die „Autor-Funktion“ begriffliche und theoretische Gestalt verleiht. Wichtig für den Autor als „,Verwalter“ von Diskursen“51 ist dabei, dass es ihm gelingt, seine ontologische Position gegenüber alternativen Positionen zu markieren und werkpolitisch zu behaupten. Matthias Schaffrick und Marcus Willand haben im Anschluss an Steffen Martus' Konzept der „Werkpolitik“52 deutlich gemacht, dass der Autor als ein „,von historischen Diskursen abhängiges Subjekt“ gedacht werden muss, deren sich dieser bemächtigt, sie sich anverwandelt oder negiert: „Das Werk erscheint [...] als Resultat eines Schreibprozesses eines Autors, der bestimmten diskursiven Herrschaftsverhältnissen unterworfen und sich dessen zumindest teilweise bewußt ist. "53 Jüngers epistolar-epistemische Autorschaft schreitet jene diskursiven Konfliktlinien ab, entlang deren Konturen sich Herrschaftsansprüche begründen

\footnotetext{
48 Steiner 2009, 95.

49 Steiner 2009, 107.

50 Foucault 1988, 21.

51 Schaffrick/Willand 2014, 34.

52 Martus 2007.

53 Schaffrick/Willand 2014, 34.
} 
und widerlegen lassen. Die Herrschaftsansprüche eines anti-darwinistischen ontologischen Denkens müssen sich dabei vom anti-ontologischen Darwinismus der modernen Biologie absetzen. In struktureller Hinsicht Pate gestanden haben dürfte hier die epistolare gelehrte Streitfragenkultur: Ein Briefpartner verteidigt gegenüber anderen seinen ,Standpunkt'. Entscheidend bei der Festlegung Jüngers auf die Rolle des Verteidigers seines Standpunktes ist allerdings die dem vormodernen Gelehrtenbrief unbekannte, bereits eingangs markierte Verbindung von Brief und Werk: Die antizipierte philologische Indienstnahme der Briefe ist bei Jünger in gleichem Maße Teil der Briefarchiv-Konzeption wie es um die schrittweise In-Werk-Setzung der Korrespondenzen geht. Philologische Indienstnahme heißt hier auch immer: Interesse an der wissensgeschichtlichen Rekonstruktion des Denkens Jüngers - und genau dieses Denken ist es auch, das schrittweise Werkförmigkeit erlangt, d.h. sich nicht mehr nur empfiehlt, als Teil eines vom eigentlichen Werk abgespaltenen Nachlasses thematisiert zu werden, sondern mit seiner Werkteleologie in das Zentrum der Auseinandersetzung mit Jünger rückt. Autorschaftsgeschichtlich betrachtet handelt es sich hierbei um die Imitation vormoderner gelehrter Autorschaft (inkl. der Rückeroberung ontologischen Wissens) unter den Bedingungen der Moderne. Genau darin besteht auch der Unterschied zur Gelehrtenbrief-Tradition: Während Jüngers epistolare Autorschaft schon immer durch spezifisch moderne werk- und nachlasspolitische Erwägungen charakterisiert ist, lässt sich der vormoderne Gelehrtenbrief - auch dort, wo er auf eine potentielle Veröffentlichung im neuen Medium der Zeitschrift hin konzipiert war ${ }^{54}$ - nicht unter die Auspizien eines aktiven autorschaftlichen Selbsthistorisierungsinteresses stellen (auch wenn es den Briefautoren gleichfalls um die Behauptung des richtigen Standpunktes ging). Im nachlasspolitischen Setting Jüngers nehmen der Brief und die vom Brief-Ich konstituierte Perspektive jedenfalls eine dreifach wichtige Funktion ein: Zum einen prägen sie den Blick auf das Gesamtarchiv (Nachlassbibliothek, Manuskripte, Geoden, Käfersammlung etc.), d.h. sie perpetuieren brieflich nachweisbare Bewertungs- und Rezeptionsmuster Jüngers im Kontext seines Nachlasses; zum anderen lassen sie sich zu jenen in den Tagebüchern gelegten Briefspuren in Bezug setzen, die bereits zu Lebzeiten des Autors erschienen. Darüber hinaus stellt die durch das Brief-Ich konstituierte Perspektive auch hinsichtlich der in den erzählenden Schriften und Essays enthaltenen epistemischnaturphilosophischen Muster eine Art Lektüreschlüssel bereit, der die Rezeption beeinflusst. Nachlass- und werkpolitisch betrachtet arbeitet Jüngers Brief-Ich an einer hermeneutischen Priorisierung, die die leserseitige „Autor-Konkretisation“ und damit die Auslegung des Gesamtwerks zu steuern sucht.

54 Vgl. zur Aufnahme von Korrespondenzen in Zeitschriften um 1700 Gierl 2004, 430-433. 


\section{Literatur}

Arnold, Heinz Ludwig (2012). Wilflinger Erinnerungen. Mit Briefen von Ernst Jünger. Göttingen. Dingler, Hugo (1943). „Die philosophische Begründung der Deszendenztheorie“, in: Die Evolution der Organismen. Ergebnisse und Probleme der Abstammungslehre. Hg. v. Gerhard Heberer. Jena: 2-19.

Dingler, Hugo (1954). „Die philosophische Begründung der Deszendenztheorie“, in: Die Evolution der Organismen. Ergebnisse und Probleme der Abstammungslehre. 2., erw. Aufl. Hg. v. Gerhard Heberer. Stuttgart: 3-24.

Fohrmann, Jürgen (2002). „,Archivprozesse“ oder über den Umgang mit der Erforschung von ,Archiv‘. Einleitung“, in: Archivprozesse. Die Kommunikation der Aufbewahrung. Hg. v. Hedwig Pompe u. Leander Scholz. Köln: 19-23.

Foucault, Michel (1988) [1969]. „Was ist ein Autor?“, in: Schriften zur Literatur. Aus d. Französischen v. Karin v. Hofer u. Anneliese Botond. Frankfurt a. M.: 7-31.

Gierl, Martin (2004). „Korrespondenzen, Disputationen, Zeitschriften. Wissensorganisation und die Entwicklung der gelehrten Medienrepublik zwischen 1670 und 1730“, in: Macht des Wissens. Die Entstehung der modernen Wissensgesellschaft. Hg. v. Richard v. Dülmen u. Sina Rauschenbach. Köln, Weimar u. Wien: 417-438.

Gutman, Mathias (2006). „Hugo Dingler und das Problem der Deszendenztheorie“, in: Wissenschaft und Leben. Philosophie in kritischer Auseinandersetzung mit Hugo Dingler. Hg. v. Peter Janich. Bielefeld: 113-122.

Heberer, Gerhard (1956). „Die Stellung Hugo Dinglers zur Evolutionstheorie“, in: Hugo Dingler. Gedenkbuch zum 75. Geburtstag. Hg. v. Wilhelm Krampf, München: 99-110.

Hesse, Hermann (2005 [1960]). „Nach der Lektüre von Ernst Jüngers Buch An der Zeitmauer“, in: Sämtliche Werke. Hg. v. Volker Michel. Bd. 20: Die Welt im Buch V. Rezensionen (1935-1962). Frankfurt a. M.: 350-353.

Jannidis, Fotis (1999). Der nützliche Autor. Möglichkeiten eines Begriffs zwischen Text und historischem Kontext, in: Rückkehr des Autors. Zur Erneuerung eines umstrittenen Begriffs. Hg. v. Fotis Jannidis, Gerhard Lauer, Matías Martínez u. Simone Winko. Tübingen: 353-389.

Jünger, Ernst u. Joseph Wulf (2019). Der Briefwechsel 1962-1974. Hg. v. Anja Keith u. Detlev Schöttker. Frankfurt a. M.

Jünger, Ernst (1982). Sämtliche Werke. Bd. 4: Strahlungen III. Tagebücher IV. Stuttgart.

Jünger, Ernst an Gerhard Heberer, 18. Juli 1956. Nachlass Ernst Jünger, DLA Marbach, Sign.: HS.1994.0009.

Kasper, Norman (2021). Episteme des „Ur“ bei Ernst Jünger. Paläontologie und Vorgeschichte. Berlin u. Boston.

Kempe, Michael (2004). „Gelehrte Korrespondenzen. Frühneuzeitliche Wissenschaftskultur im Medium postalischer Kommunikation“, in: Die Medien der Geschichte. Historizität und Medialität in interdisziplinärer Perspektive. Hg. v. Fabio Crivellari, Kay Kirchmann, Marcus Sandl u. Rudolf Schlögl. Konstanz: 407-430.

Martus, Steffen (2007). Werkpolitik. Zur Literaturgeschichte kritischer Kommunikation vom 17. bis ins 20. Jahrhundert mit Studien zu Klopstock, Tieck, Goethe und George. Berlin u. New York.

Morat, Daniel (2014). Persönliche Konstellationen. Ernst Niekisch, in: Ernst Jünger-Handbuch. Hg. v. Matthias Schöning. Stuttgart: 389-396. 
Morat, Daniel (2012). „Die Entpolitisierung des Politischen. Ernst Jüngers Essayistik der 1950er Jahre“, in: Ernst Jünger und die Bundesrepublik. Ästhetik - Politik - Zeitgeschichte. Hg. v. Matthias Schöning u. Ingo Stöckmann. Berlin u. New York: 163-183.

Müller-Tamm, Jutta (2014). „Die Denkfigur als wissensgeschichtliche Kategorie“, in: WissensOrdnungen. Zu einer historischen Epistemologie der Literatur. Hg. v. Nicola Gess u. Sandra Janßen. Berlin u. New York: 100-120.

Neumann, Michael (2014). „Nachlasspublikationen. Briefwechsel mit Gerhard Nebel“, in: Ernst Jünger-Handbuch. Hg. v. Matthias Schöning. Stuttgart: 298-302.

Schaffrick, Matthias u. Marcus Willand (2014). „Autorschaft im 20. Jahrhundert. Bestandsaufnahme und Positionsbestimmung“, in: Theorien und Praktiken der Autorschaft. Hg. v. Matthias Schaffrick u. Marcus Willand. Berlin u. New York: 3-148.

Schöttker, Detlev (2016). „Korrespondenz und Nachleben. Ernst Jüngers Briefarchiv“, in: Ränder des Archivs. Kulturwissenschaftliche Perspektiven auf das Entstehen und Vergehen von Archiven. Hg. v. Falko Schmieder u. Daniel Weidner. Berlin: 111-133.

Schuster, Jörg u. Jochen Strobel (2012). „Briefe und Interpretationen. Über Möglichkeiten zu einer Geschichte der Briefkultur und über die Möglichkeit kulturhistorischer Skizzen mittels Brieflektüren“, in: Briefkultur. Texte und Interpretationen - von Martin Luther bis Thomas Bernhard. Hg. v. Jörg Schuster u. Jochen Strobel. Berlin u. New York: XI-XXIV.

Sina, Kai (2017). „Die vergangene Zukunft der Literatur. Zeitstrukturen und Nachlassbewusstsein in der Moderne“, in: Nachlassbewusstsein. Literatur, Archiv, Philologie 1750-2000. Hg. v. Kai Sina u. Carlos Spoerhase. Göttingen 2017: 49-74.

Sina, Kai u. Carlos Spoerhase (2013). „Nachlassbewusstsein. Zur literaturwissenschaftlichen Erforschung seiner Entstehung und Entwicklung“, in: Zeitschrift für Germanistik 23,3: 607-623.

Spoerhase, Carlos (2007). Autorschaft und Interpretation. Methodische Grundlagen einer philologischen Hermeneutik. Berlin u. New York.

Steiner, Felix (2009). Dargestellte Autorschaft. Autorkonzept und Autorsubjekt in wissenschaftlichen Texten. Tübingen.

Trawny, Peter (2014). „Persönliche Konstellationen. Martin Heidegger“, in: Ernst JüngerHandbuch. Hg. v. Matthias Schöning. Stuttgart: 368-371.

van de Löcht, Joana (2020). [Rez.] Sibylle Benninghoff-Lühl: Die ganze Welt ein Garten? Flora und Fauna in Ernst Jüngers schriftlichem Nachlass, in: Jünger Debatte 3: 227-230.

Vellusig, Robert (2018). „Die Poesie des Briefs. Eine literaturanthropologische Skizze“, in: Was ist ein Brief? Aufsätze zu epistolarer Theorie und Kultur / What is a letter? Essays on epistolary theory and culture. Hg. v. Marie Isabel Matthews-Schlinzig u. Caroline Socha. Würzburg 2018: 57-75.

Vellusig, Robert (2020). „Literarische Anthropologie und Brief“, in: Handbuch Brief. Von der Frühen Neuzeit bis zur Gegenwart. Hg. v. Marie Isabel Matthews-Schlinzig, Jörg Schuster, Gesa Steinbrink u. Jochen Strobel. Bd. 1: Interdisziplinarität - Systematische Perspektiven Briefgenres. Berlin u. Boston: 203-221.

Villinger, Ingeborg (2014). „Nachlasspublikationen. Briefwechsel mit Carl Schmitt“, in: Ernst Jünger-Handbuch. Hg. v. Matthias Schöning. Stuttgart: 293-297.

Vodička, Felix (1976). „Die Konkretisation des literarischen Werks. Zur Problematik der Rezeption von Nerudas Werk“ [1941], in: Felix Vodička: Die Struktur der literarischen Entwicklung. Hg. v. der Forschungsgruppe für strukturale Methoden in der Sprach- und Literaturwissenschaft an der Universität Konstanz. Aus dem Tschechischen v. Christian Tuschinsky, Peter Richter u. Frank Boldt. München: 87-125. 
Wallnig, Thomas (2020). „Gelehrtenbriefe“, in: Handbuch Brief. Von der Frühen Neuzeit bis zur Gegenwart. Hg. v. Marie Isabel Matthews-Schlinzig, Jörg Schuster, Gesa Steinbrink u. Jochen Strobel. Bd. 1: Interdisziplinarität - Systematische Perspektiven - Briefgenres. Berlin u. Boston: 471-483.

Willand, Marcus (2011). „Autorfunktionen in literaturwissenschaftlicher Theorie und interpretativer Praxis. Eine Gegenüberstellung“, in: Journal of Literary Theory 5: 279-301.

Winko, Simone (2002). „Autor-Funktionen. Zur argumentativen Verwendung von Autorkonzepten in der gegenwärtigen literaturwissenschaftlichen Interpretationspraxis“, in: Autorschaft. Positionen und Revisionen. Hg. v. Heinrich Detering. Stuttgart u. Weimar: 334-354. 